\title{
A INFLUÊNCIA DA TERMOTERAPIA NO GANHO DE FLEXIBILIDADE DOS MÚSCULOS ISQUIOTIBIAIS
}

\author{
THE INFLUENCE OF THERMOTHERAPY IN THE FLEXIBILITY GAIN OF THE HAMSTRING MUSCLES
}

LA INFLUENCIA DE LA TERMOTERAPIA EN LA FLEXIBILIDAD DE LOS MÚSCULOS ISQUIOTIBIALES

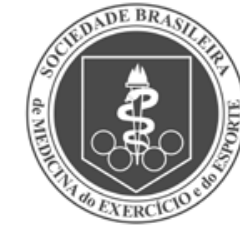

Artigo Original

Original ARTICLE

Artículo Original
José Herivelton Cardoso de Andrade Filho ${ }^{1}$ (Fisioterapeuta) Thales Costa e Silva do Espírito Santo ${ }^{1}$ (Fisioterapeuta)

Samara Géssica Germano Facó (Fisioterapeuta)

Alessandra Tanuri Magalhães' (Fisioterapeuta)

Baldomero Antonio Kato da Silva (Fisioterapeuta)

Barbara Visiglia Minghini

(Fisioterapeuta)

Cristiano Sales da Silva'

(Fisioterapeuta)

Vinícius Saura Cardoso

(Fisioterapeuta)

1. Universidade Federal do Piauí (UFPI), Parnaíba, Piauí, Brasil.

\section{Correspondência:}

Vinicius Saura Cardoso

BR 343, 4228, casa 16, São Judas

Tadeu, Parnaíba, Piauí, Brasil. 64206-260.

vscfisio@ufpi.edu.br

\section{RESUMO}

Introdução: A flexibilidade muscular é descrita como a capacidade do músculo realizar a extensão de suas fibras, influenciando a mobilidade articular. A diatermia aplicada à musculatura prepara o corpo para atividades físicas, por acelerar o metabolismo das fibras musculares e diminuir a resistência intramuscular, aumentando assim a variedade de movimento e a eficiência mecânica. A crioterapia tem diversas funções fisiológicas quando aplicada ao corpo humano, as quais, em conjunto, propiciam o relaxamento muscular. Objetivo: Avaliar o comportamento da flexibilidade dos músculos isquiotibiais frente à aplicação de alongamento passivo associado a termoterapias quente e fria. Métodos: Este estudo foi composto de 24 voluntários, de ambos os sexos, distribuídos aleatoriamente em três grupos, sendo eles: grupo alongamento passivo (G1), grupo crioterapia precedendo alongamento passivo (G2) e grupo diatermia precedendo alongamento passivo (G3). Os voluntários foram submetidos a avaliações de amplitude de movimento através da goniometria da flexão de quadril com o joelho estendido, sendo uma avaliação inicial realizada antes de iniciar o protocolo e reavaliações ao final de cada uma das quatro semanas de intervenção. Resultados: Verificou-se aumento significativo da flexão de quadril no G2 e G3 com relação ao G1. Não houve diferença estatística entre G2 e G3. Conclusão: O uso da diatermia, assim como da crioterapia, associado ao alongamento passivo é favorável para o ganho de flexibilidade muscular e, consequentemente, de amplitude de movimento em jovens saudáveis.

Descritores: exercícios de alongamento muscular; crioterapia; diatermia; amplitude de movimento articular.

\section{ABSTRACT}

Introduction: Muscle flexibility is described as the ability of the muscle to extend its fibers, influencing joint mobility. Diathermy applied to the muscles prepares the body for physical activity, by accelerating the metabolism of the muscle fibers and decreasing the intramuscular resistance, thereby increasing the range of motion and the mechanical efficiency. Cryotherapy has various physiological functions when applied to the human body, which, together, provide muscle relaxation. Objective: To evaluate the flexibility of hamstring muscles by applying passive stretching associated with hot and cold thermotherapy. Methods: This study consisted of 24 volunteers of both sexes randomly divided into three groups: passive stretching group (G1), cryotherapy preceding passive stretching group (G2) and diathermy preceding passive stretching group (G3). The volunteers were evaluated for range of motion by goniometry of hip flexion with the knee extended, with an initial assessment carried out before starting the protocol and revaluations at the end of each of the four weeks of intervention. Results: There was significant increase in hip flexion in G2 and G3 compared to G1. There was no statistical difference between G2 and G3. Conclusion: The use of diathermy and cryotherapy associated with passive stretching is favorable for increasing muscular flexibility and hence the amplitude of motion in healthy young adults.

Keywords: muscle stretching exercise; cryotherapy; diathermy, range of motion, articular.

\section{RESUMEN}

Introducción: La flexibilidad muscular se describe como la capacidad del músculo para lograr la extensión de sus fibras, que afecta a la movilidad articular. La diatermia aplicada a los músculos prepara el cuerpo para la actividad física, mediante la aceleración del metabolismo de las fibras musculares y disminución de la resistencia intramuscular, lo que aumenta el rango de movimiento y la eficiencia mecánica. La crioterapia tiene diversas funciones fisiológicas cuando es aplicada al cuerpo humano, que, en conjunto, proporcionan la relajación muscular. Objetivo: Evaluar la flexibilidad de los músculos isquiotibiales frente a la aplicación de alongamiento pasivo asociado a termoterapia caliente y fría. Métodos: Este estudio se compone de 24 voluntarios de ambos sexos divididos aleatoriamente en tres grupos, a saber: grupo de estiramiento pasivo (G1), grupo de crioterapia antes del estiramiento pasivo (G2) y grupo de diatermia antes del estiramiento pasivo (G3). Los voluntarios fueron sometidos a evaluaciones de amplitud de movimiento a través de la goniometría de la flexión de cadera con la rodilla extendida, siendo una evaluación inicial realizada antes de iniciar el protocolo y reevaluaciones al final de cada una de las cuatro semanas de intervención. Resultados: No hubo aumento significativo de la flexión de la cadera en G2 y G3 en comparación con G1. No hubo diferencia estadística entre G2 y G3. Conclusión: El uso de diatermia y crioterapia asociado con el estiramiento pasivo es favorable para aumentar la flexibilidad muscular y por lo tanto el rango de movimiento en adultos jóvenes sanos.

Descriptortes: ejercicios de estiramiento muscular; crioterapia; diatermia; rango del movimiento articular. 


\section{INTRODUÇÃO}

O tecido muscular esquelético tem como característica ser flexível, influenciando a mobilidade articular. Desta forma, a diminuição ou aumento da flexibilidade afetará de forma direta a amplitude de movimento $(\mathrm{AM})^{1,2}$, podendo modificar atividades de vida diária (AVD), atividades laborais, bem estar psicológico e o desempenho esportivo ${ }^{3-5}$. Desta forma, profissionais de saúde defendem o ganho de flexibilidade para a melhora do desempenho em AVD e atividades esportivas de alto rendimento ${ }^{4}$.

O treinamento para ganho de flexibilidade proporciona ao grupo muscular aumento das capacidades físicas de rendimento e suporte ao esforço, economia do trabalho muscular, manutenção da postura correta, facilitação da aprendizagem motora, maior potencial de recuperação após atividade física e conservação da autonomia nas atividades habituais ${ }^{6}$. Entre os atletas, a flexibilidade propicia ainda condições para desenvolver agilidade, velocidade e força, além de minimizar o risco de lesões 7,8 .

Fisioterapeutas frequentemente avaliam a flexibilidade dos tecidos de seus pacientes, por meio da mensuração da AM, e utilizam técnicas de alongamento para restaurar a mobilidade normal, quando necessário9-11. Estas técnicas promovem a flexibilidade por aumentar a extensibilidade musculotendínea e do tecido conjuntivo periarticular, favorecendo maior AM. Além disso, as propriedades viscosas dos tecidos fazem com que este ganho não seja imediatamente reversível, fato que implica em maior funcionalidade aos indivíduos submetidos a estes exercícios ${ }^{11-13}$.

Entretanto, ainda não há consenso sobre como realizar o alongamento de forma que haja a máxima eficácia no ganho de flexibilidade. Assim, faz-se necessário o aprimoramento dos protocolos usados durante o alongamento, sendo este, atualmente, um dos grandes objetivos de profissionais como fisioterapeutas, médicos desportivos e educadores físicos ${ }^{14,15}$. Os estudos de Brasileiro et al. ${ }^{16}$ e Silva et al. ${ }^{17}$ tiveram por objetivo a avaliação dos efeitos da associação de termoterapia quente e fria ao alongamento, sugerindo novos estudos sobre o tema devido à escassez de publicações correlatas.

A termoterapia com aplicação de calor aumenta o suprimento sanguíneo, acelera o metabolismo das fibras musculares e reduz a resistência intramuscular ${ }^{10,18}$. A diatermia por ondas curtas (OC) é um recurso terapêutico de relevante eficácia no aquecimento de tecidos profundos, por meio de radiação eletromagnética ${ }^{19}$, aumentando a temperatura entre $4^{\circ}$ e $5^{\circ}$ numa profundidade de $3 \mathrm{~cm}^{20,21}$, sendo necessária a manutenção desta temperatura por um período de cerca de 5 minutos para um aumento significante na extensibilidade dos tecidos ${ }^{20}$.

Já a crioterapia é definida como a aplicação terapêutica de qualquer substância ao corpo que resulte em remoção de calor corporal, reduzindo, assim a temperatura dos tecidos ${ }^{22}$. Esta tem diversas funções fisiológicas quando aplicada no corpo humano, dentre elas, diminuição na velocidade de condução nervosa, liberação de endorfinas, redução da atividade do fuso muscular e da força tênsil, propiciando o relaxamento muscular ${ }^{23}$. Geralmente, é necessário aplicação deste recurso durante cinco a 15 minutos para que sejam obtidos os efeitos produzidos pelo resfriamento ${ }^{24}$.

Tanto o uso da crioterapia quanto da terapia com calor como subsídio ao alongamento pode favorecer ao ganho de flexibilidade, entretanto, agindo de diferentes formas ${ }^{16,17}$. O objetivo deste estudo foi avaliar o comportamento da flexibilidade dos músculos isquiotibiais frente à aplicação de alongamento passivo, associado à termoterapia quente e fria, durante quatro semanas.

\section{MATERIAIS E MÉTODOS}

Participaram do estudo 24 voluntários saudáveis com idade média de 20,18 1 1,18 anos, de ambos os sexos (12 homens e 12 mulheres) e cujo membro inferior dominante referido foi o direito. Os voluntários não possuíam histórico de doenças ortopédicas e neurológicas e foram distribuídos aleatoriamente em três grupos de mesmo tamanho, sendo estes: grupo alongamento passivo (G1), grupo crioterapia mais alongamento passivo (G2) e grupo diatermia mais alongamento passivo (G3). Os voluntários foram informados sobre a pesquisa e aceitaram participar assinando o Termo de Consentimento Livre e Esclarecido, de acordo com a Resolução 466/12.

O trabalho foi aprovado pelo Comitê de Ética e Pesquisa da Universidade Federal do Piauí (parecer 727.689).

\section{Procedimento}

A avaliação da AM da flexão do quadril com o joelho estendido foi realizada utilizando um goniômetro universal (FisioStore') e o voluntário foi posicionado em decúbito dorsal, com corpo estabilizado através de faixas para evitar compensação durante o movimento. Uma faixa estabilizadora foi fixada na coxa do membro inferior que não estava sendo avaliado e a outra faixa colocada ao nível das cristas ilíacas para estabilização do quadril. A AM de flexão do quadril relaciona-se diretamente com o posicionamento do joelho, isso se deve a presença de músculos biarticulares localizados posteriormente à articulação do quadril (isquiotibiais). Diante disto, durante a avaliação, o joelho do voluntário foi estabilizado pelo avaliador ${ }^{25}$. Foram realizadas cinco avaliações da AM do quadril, as quatro primeiras no início de cada semana do protocolo, antes das sessões de alongamento, e a quinta avaliação dois dias após a última sessão.

Os grupos foram submetidos a sessões de alongamento passivo, compostas por três séries de trinta segundos de alongamento dos músculos isquiotibiais com dez segundos de repouso entre as séries. O voluntário foi posicionado em decúbito dorsal, com o quadril estabilizado e o terapeuta realizou a flexão de quadril com o joelho estendido ao máximo, até o ponto em que o mesmo referiu desconforto, obtendo-se o alongamento muscular nesta posição. Este protocolo foi proposto por Bandy et al. ${ }^{1}$.

No G1 foi realizado o protocolo de alongamento passivo acima descrito com frequência de três sessões semanais durante quatro semanas. No G2 foi aplicada crioterapia, por meio de bolsa térmica, no terço médio da região posterior da coxa para resfriamento, durante 15 minutos, logo após foi realizado o protocolo de alongamento passivo. No G3 foi realizada aplicação de OC (calor profundo), modelo Thermopulse Compact (Ibramed ${ }^{\circ}$ ), para aquecimento durante $15 \mathrm{mi}$ nutos, com as placas dispostas em posição coplanar no terço médio da região posterior da coxa e intensidade de $80 \mathrm{~W}^{16}$. Logo após foi realizado o protocolo de alongamento passivo. Desta forma, todos os grupos tiveram a frequência de três intervenções terapêuticas durante quatro semanas. O protocolo foi efetuado em ambos os membros inferiores, entretanto, somente foi adotada a avaliação do membro inferior direito (MID) na análise no estudo.

\section{Análise Estatística}

As comparações intragrupos tiveram periodicidade semanal, e foram realizadas utilizando-se o teste $t$ de Student para amostras relacionadas.

Para comparação intergrupos foram consideradas as medias das diferenças entre a última e a primeira avaliação para cada grupo, sendo utilizada Análise de Variância (ANOVA) com post hoc test de Tukey. O nível de significância considerado foi de 95\% ( $p<0,05)$.

\section{RESULTADOS}

Todos os voluntários concluíram o estudo. Ao avaliar a AM nos três grupos, pode-se verificar aumento, representado pelo ganho em graus, significativo da flexão de quadril no G2 e G3 em relação ao G1. Quando comparado o G2 com o G3 não houve diferença significativa (Figura 1). 
Analisando cada grupo separadamente pode-se observar que o ganho de AM nos grupos estudados foi significativo a partir da $3^{a}$ semana (4a avaliação) tendo como referência a avaliação inicial. Para o G1 pode-se observar um incremento de $24,30 \%$ da AM ao final do estudo (Figura 2). O G2 obteve o ganho de 36,53\% (Figura 3) e para o G3 houve aumento da AM em 40,16\% (Figura 4).

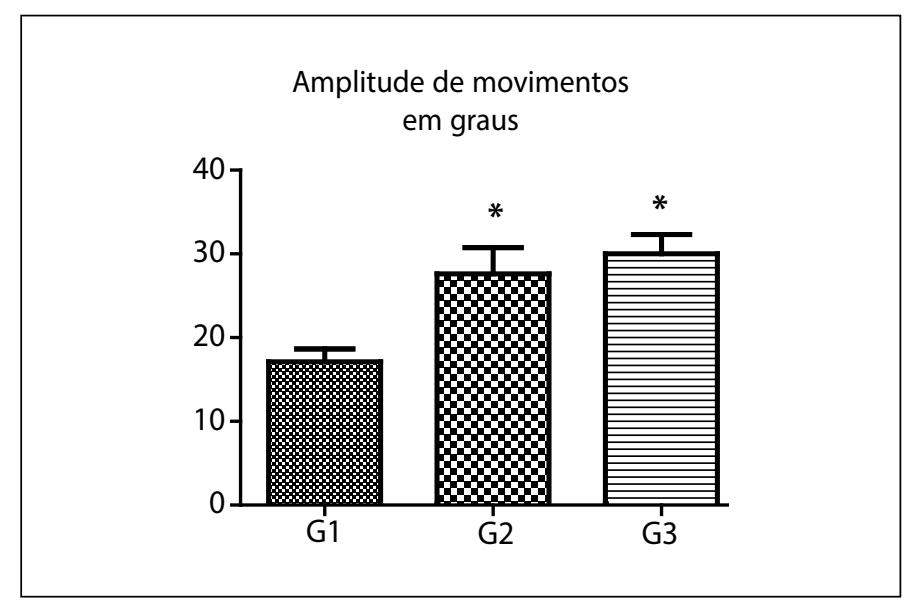

Figura 1. Comparação da AM (final-inicial) da flexão do quadril (em graus), nos grupos 01 (alongamento), 02 (crioterapia + alongamento) e 03 (ondas curtas + alongamento). $(\mathrm{P}<0,05)$.

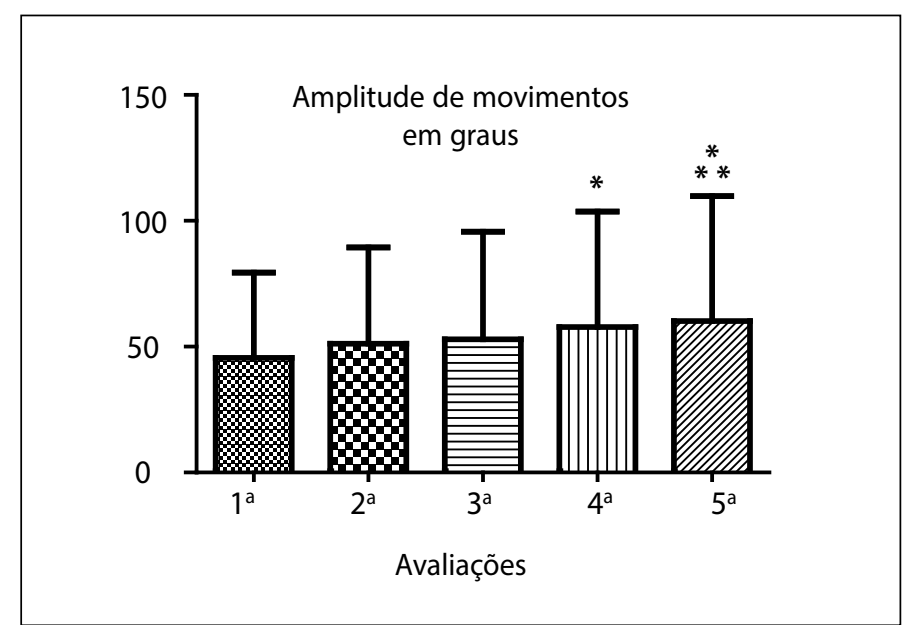

Figura 2. Medida, em graus, da AM da flexão de quadril do G1 durante o estudo apresentando diferença estatística a partir da quarta semana tendo como referencia a primeira semana. $(P<0,05)$.

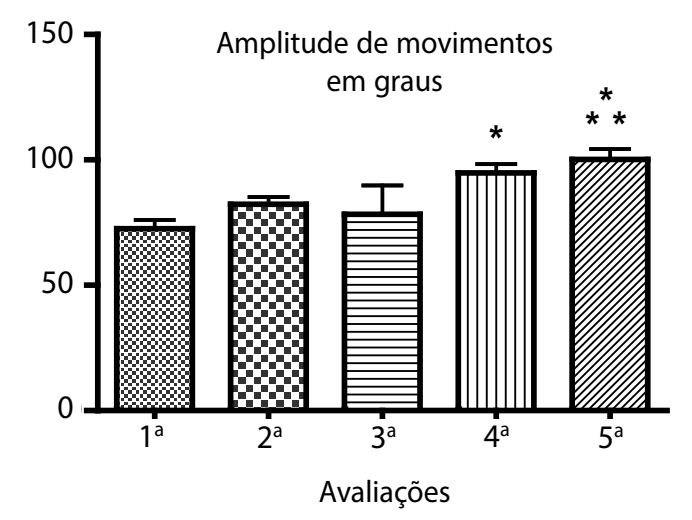

Figura 3. Medida, em graus, da AM da flexão de quadril do G2 durante o estudo apresentando diferença estatística a partir da quarta semana tendo como referencia a primeira semana. $(P<0,05)$.

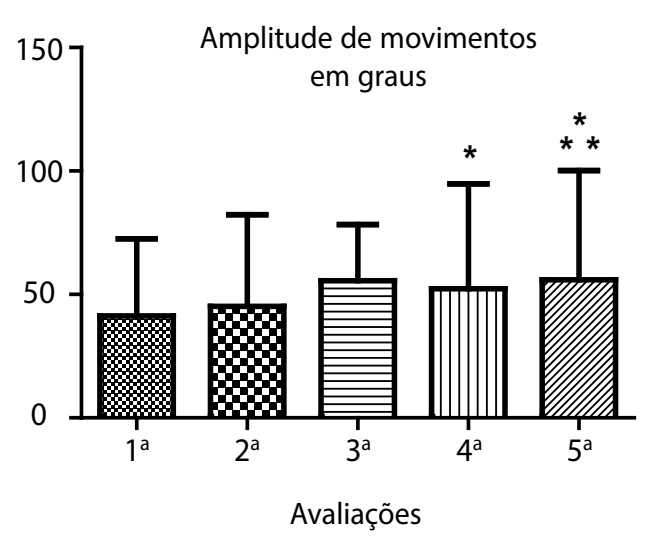

Figura 4. Medida, em graus, da AM da flexão de quadril do G3 durante o estudo apresentando diferença estatística a partir da quarta semana tendo como referencia a primeira semana. $(P<0,05)$.

\section{DISCUSSÃO}

Os músculos isquiotibiais desempenham papel relevante tanto na eficácia, como na eficiência dos movimentos humanos básicos, como caminhada e corrida. Na prática clínica têm-se observado que a limitação de flexibilidade deste grupo muscular é comum na população em geral. Da mesma forma, na área esportiva, isquiotibiais encurtados têm sido relacionados a tensões musculares, desenvolvimento de tendinopatia patelar e dor femoropatelar, dano muscular após o exercício excêntrico e redução no desempenho esportivo ${ }^{26,27}$. Neste sentido, foi observado que o alongamento passivo de vários grupos musculares, principalmente dos isquiotibiais, melhora o comprimento e a extensibilidade muscular, tanto de modo imediato, como em efeito em longo prazo do presente treinamento ${ }^{28}$.

O estudo averiguou que os três grupos em avaliação tiveram eficiente aumento na flexibilidade dos músculos isquiotibiais após os protocolos adotados. O G3 (diatermia + alongamento) apresentou diferença significante em relação ao G1 (alongamento), divergindo dos estudos de Brasileiro et al. ${ }^{16}$ e Silva et al. ${ }^{17}$, que também utilizaram a diatermia por ondas curtas associada ao alongamento para ganho de flexibilidade dos músculos isquiotibiais, porém sem alterações significativas na AM.

O resultado do presente estudo vem de acordo com a prática clínica que utiliza técnicas de alongamento associadas ao calor para obter um maior ganho da flexibilidade. $\mathrm{O}$ aquecimento aumenta o suprimento de sangue e a liberação do oxigênio da mioglobina e hemoglobina para os músculos. Estas mudanças aceleram o metabolismo das fibras musculares e diminuem a resistência intramuscular, aumentando assim a eficiência mecânica do movimento ${ }^{10,18}$.

O G2 (crioterapia + alongamento) também apresentou diferença significante em relação ao G1. É fato que a diminuição de dor e condução nervosa é ocasionada pelo resfriamento ${ }^{16,29-32}$, o que traz consequências diretas para as manobras de alongamento. A limitação álgica durante as manobras de alongamento precede a limitação tecidual, desta forma, a sensação subjetiva de desconforto na musculatura que está sendo alongada pode reduzir a eficiência da manobra, minimizando possíveis alterações viscoelásticas nos tecidos como a diminuição de flexibilidade das proteínas musculares quando resfria$\mathrm{do}^{33}$. Uma vez que o sujeito aumenta a sua tolerância às manobras com o resfriamento, maior alongamento seria permitido. Em relação a diminuição da condução nervosa, estudos anteriores que utilizaram o relaxamento muscular obtido com a aplicação da crioterapia, sugerem que a diminuição na tensão muscular ocorre devido a redução na frequência de disparo dos fusos musculares ${ }^{34,35}$. 
Na clínica de fisioterapia, o uso da crioterapia para ganho de AM, ainda é questionada, pois esta técnica desencadeia respostas fisiológicas adversas sobre as propriedades viscoelásticas dos tecidos. Knight 22 , afirma que a presença de hipotermia aumenta a rigidez do tecido conjuntivo, diminuindo sua extensibilidade, o que sugere que a associação entre resfriamento e alongamento seja prejudicial quando o objetivo é promover aumento da extensibilidade deste tecido.

Fisiologicamente, para pacientes em fase aguda e/ou apresentando quadro álgico significante é recomendada a utilização de termoterapia fria ou crioterapia, devido seus efeitos analgésicos e de redução de respostas inflamatórias, além de diminuir o desconforto durante as sessões de alongamento ${ }^{36}$, comum nos pacientes com maior restrição de movimento (início do tratamento). Já em quadros crônicos e/ou quando o indivíduo apresenta-se em tratamento ativo, a termoterapia por meio de calor é a melhor indicada, por seus efeitos de potencialização da atividade metabólica e promoção de relaxamento muscular ${ }^{37}$. Desta forma, sugere-se o uso de crioterapia associada ao alongamento passivo quando pacientes apresentam indicação de ganho de AM e se encontram na fase aguda do processo inflamatório. O uso do OC associado ao alongamento passivo seria indicado para pacientes com indicação de ganho de AM e se encontram na fase crônica do processo inflamatório.
Pode-se observar, quando comparado intragrupos, que todos os grupos apresentaram acréscimo na AM, porém este aumento foi estatisticamente significante a partir da terceira semana. Isso pode ser atribuído ao fato da musculatura ter um tempo fisiológico de adaptação ao estresse causado pelo alongamento. Segundo Herbert ${ }^{38}$, Hall e Brod ${ }^{39}$ os efeitos do alongamento podem ser divididos em agudos e crônicos. Os agudos ou imediatos são resultado da flexibilidade do componente elástico da unidade musculotendínea. Desta forma, o OC associado ao alongamento passivo pode auxiliar nos efeitos imediatos, pois modifica a elasticidade das proteínas musculares ${ }^{40}$. Já os efeitos crônicos resultam em remodelamento adaptativo da estrutura muscular ${ }^{38,39}$.

\section{CONCLUSÃO}

Os resultados deste estudo sugerem que o uso da termoterapia quente, por ondas curtas, e fria, associadas ao alongamento passivo, é favorável para o ganho de flexibilidade muscular e, consequentemente, da AM em jovens saudáveis.

Todos os autores declararam não haver qualquer potencial conflito de interesses referente a este artigo.

CONTRIBUIÇÕES DOS AUTORES: Todos os autores contribuíram individualmente e de forma significante para a realização do manuscrito. JHCAF(0000-0001-7329-7894)*, TCSES(0000-0002-6140-1268)* e SGGF (0000-0003-3242-1419)* fizeram as avaliações e aplicaram o protocolo do estudo. BAKS $(0000-0001-9337-9563)^{*}$ e CSS (0000-0002-2823-7064)* realizaram as análises estatísticas do estudo. ATM (0000-0003-0647-3506)* e BVM (000-0002-3827-1047)* realizaram a revisão de literatura para publicação e VSC (0000-0003-0269-7473)* idealizou o projeto, realizou o acompanhamento das coletas e organizou o artigo. Todos os autores contribuíram com o conceito intelectual e revisaram o artigo antes da submissão. *Numero ORCID (Open Researcher and Contributor ID).

\section{REFERÊNCIAS}

1. Bandy WD, Irion JM, Briggler M. The effect of time and frequency of static stretching on flexibility of the hamstring muscles. Phys Ther. 1997;77(10):1090-6.

2. Veiga PHA, Daher CRM, Morais MFF. Alterações posturais e flexibilidade da cadeia posterior nas lesões em atletas de futebol de campo. Rev Bras Ciênc Esporte. 2011;33(1):235-48.

3. Almeida TT, Jabur MN. Mitos e verdades sobre flexibilidade: reflexões sobre o treinamento de flexibilidade na saúde dos seres humanos. Motricidade. 2007;3(1):337-44.

4. Depino GM, Webright WG, Arnold BL. Duration of maintained hamstring flexibility after cessation of an acute static stretching protocol. J Athl Train. 2000;35(1):56-9.

5. Johnson AW, Mitchell UH, Meek K, Feland JB. Hamstring flexibility increases the same with 3 or 9 repetitions of stretching held for a total time of 90 s. Phys Ther Sport. 2014;15(2):101-5.

6. Theodoro PFR, Salve MGC. Análise da flexibilidade em mulheres trabalhadoras. Rev Mov Percepção. 2005; 5(7):116-23

7. Silva RG. Análise da flexibilidade em idosas praticantes de caminhada do município de Montes Claros, MG. Rev Cons Ext. 2008;1(1):32-9.

8. Zakas A. The effect of stretching duration on the lower-extremity flexibility of adolescent soccer players. J Bodyw Mov Ther. 2005;9:220-5.

9. Youdas JW, Krause DA, Egan KS, Therneau TM, Laskowski ER. The effect of static stretching of the calf muscle-tendon unit on active ankle dorsiflexion range of motion. J Orthop Sports Phys Ther. 2003;33(7):408-17.

10. Thacker SB, Gilchrist J, Stroup DF, Kimsey CD Jr. The impact of stretching on sports injury risk: a systematic review of the literature. Med Sci Sports Exerc. 2004;36(3):371-8.

11. Aquino CF, Fonseca ST, Gonçalves GG, Silva PL, Ocarino JM, Mancini MC. Stretching versus strength training in lengthened position in subjects with tight hamstring muscles: a randomized controlled trial. Man Ther. 2010;15(1):26-31.

12. Alencar TAM, Matias KFS. Princípios fisiológicos do aquecimento e alongamento muscular na atividade esportiva. Rev Bras Med Esporte. 2010; 6(3):230-4.

13. Voigt L, Vale RGS, Abdala DW, Freitas WZ, Novaes JS, Dantas EHM. Efeitos de três repetições de 10 segundos de estímulo do método estático para o desenvolvimento da flexibilidade de homens adultos jovens. Rev Bras Ciênc Esporte. 2010;32(2-4):155-66.

14. Vieira WHB, Valente RZ, Andrusaitis FR, Greve JMA, Brasileiro JS. Efeito de duas técnicas de alongamento muscular dos ísquios-tibiais na amplitude de extensão ativa do joelho e no pico de torque. Rev Bras Fisioter. 2005;9:71-6.

15. Herbert RD, Gabriel M. Effects of stretching before and after exercising on muscle soreness and risk of injury: systematic review. BMJ. 2002;325(7362):468.

16. Brasileiro JS, Faria AF E Queiroz LL. Influencia do resfriamento e do aquecimento local na flexibilidade dos músculos isquiotibiais. Rev Bras Fisioter. 2007;11(1):57-61.

17. Silva SA, Oliveira DJ, Jaques MJN, Araújo RC. Efeito da crioterapia e termoterapia associados ao alongamento estático na flexibilidade dos músculos isquiotibiais. Motricidade. 2010;6(4):55-62.

18. Hurley MV, Bearne LM. Non-exercise physical therapies for musculoskeletal conditions. Best Pract Res Clin Rheumatol. 2008;22(3):419-33.

19. Assunção MLA, Ramos AAT, Lima BA. Termoterapia profunda como tratamento fisioterapêutico na osteoartrite. Rev Ciênc Méd. 2010;19(1-6):73-9.

20. Robertson VJ, Ward AR, Jung P. The effect of heat on tissue extensibility: a comparison of deep and superficial heating. Arch Phys Med Rehabil. 2005;86(4):819-25.
21. Draper DO, Knight K, Fujiwara T, Castel JC. Temperature change in human muscle during and after pulsed short-wave diathermy. J Orthop Sports Phys Ther. 1999;29(1):13-8.

22. Knight KL. Crioterapia no tratamento das lesões esportivas. São Paulo: Manole; 2000.

23. Pangarkar S, Lee PC. Conservative treatment for neck pain: medications, physical therapy, and exercise Phys Med Rehabil Clin N Am. 2011;22(3):503-20.

24. Mortari DM, Mânica AP, Pimentel GL. Efeitos da crioterapia e facilitação neuromuscular proprioceptiva sobre a força muscular nas musculaturas flexora e extensora de joelho. Fisioter Pesq. 2009;16(4):329-34

25. Sacco ICN, Tanaka C. Cinesiologia e biomecânica dos complexos articulares. Rio de Janeiro: Guanabara Koogan; 2008.

26. Ayala F, Sainz de Baranda P, De Ste Croix M, Santonja F. Comparison of active stretching technique in males with normal and limited hamstring flexibility. Phys Ther Sport. 2013;14(2):98-104.

27. Castellote-Caballero Y, Valenza MC, Martín-Martín L, Cabrera-Martos I, Puentedura EJ, Fernández-de-Las-Peñas C. Effects of a neurodynamic sliding technique on hamstring flexibility in healthy male soccer players. A pilot study. Phys Ther Sport. 2013;14(3):156-62.

28. Smith M, Fryer G. A comparison of two muscle energy techniques for increasing flexibility of the hamstring muscle group. J Bodyw Mov Ther. 2008;12(4):312-7.

29. Fukuchi CA, Duarte M, Stefanyshyn DJ. Postural sway following cryotherapy in healthy adults. Gait Posture. 2014;40(1):262-5.

30. Espinoza HJG, Bustamante IPL, Pérez SJM. Revisión sistemática sobre el efecto analgésico de la crioterapia en el manejo del dolor de origen músculo esquelético. Rev Soc Esp Dolor. 2010;17(5):242-52

31. Zhang J, Pan T, Wang JH. Cryotherapy suppresses tendon inflammation in an animal model. J Orthop Translat. 2014;2(2):75-81.

32. Ascensão A, Leite M, Rebelo AN, Magalhäes S, Magalhäes J. Effects of cold water immersion on the recovery of physical performance and muscle damage following a one-off soccer match. J Sports Sci. 2011;29(3):217-25

33. Halbertsma JP, van Bolhuis Al, Göeken LN. Sport stretching: effect on passive muscle stiffness of shor hamstrings. Arch Phys Med Rehabil. 1996;77(7):688-92.

34. Swenson C, Swärd L, Karlsson J. Cryotherapy in sports medicine. Scand J Med Sci Sports. 1996;6(4):193-200.

35. Eston R, Peters D. Effects of cold water immersion on the symptoms of exercise-induced muscle damage. J Sports Sci. 1999;17(3):231-8.

36. Busarello FO, Souza FT, Paula GF, Vieira L, Nakayama GK, Bertolini GRF. Ganho de extensibilidade dos músculos isquiotibiais comparando o alongamento estático associado ou não à crioterapia. Fisiote Mov. 2011;24(2):247-54.

37. Boldrini FC, Lopes AD, Liebano RE. Efeitos da diatermia por ondas curtas no torque do músculo quadríceps femoral durante a estimulação elétrica neuromuscular e contração voluntária em indivíduos saudáveis. Rev Bras Med Esporte. 2013;19(4):247-51.

38. Herbert R. The passive mechanical properties of muscle and their adaptations to altered patterns of use. Aust J Physiother. 1988;34(3):141-9.

39. Hall CM, Brod LT. Exercícios terapêuticos na busca da função. 2a ed. Rio de Janeiro: Guanabara Koogan; 2007 40. Signori LU, Voloski FRS, Kerkhoff AC, Brignoni L, Plentz RDM. Efeito de agentes térmicos aplicados previamente a um programa de alongamentos na flexibilidade dos músculos isquiotibiais encurtados. Rev Bras Med Esporte. 2008;14(4):328-31. 